\title{
THERMAL INDUCED CLUSTERING OF POLYSILICON FILM ON SILICON NITRIDEISILICON DIOXIDE BED ON SILICON SUBSTRATE
}

\author{
Shobha Kanta Lamichhane* \\ *Tribhuvan University, Prithwi Narayan Campus, Pokhara, Nepal.
}

\begin{abstract}
Polysilicon (PS) grains are clustered in an order in the presence of thermal doping of boron in low pressure chemical vapour deposition (LPCVD). PS layer is lying on Silicon Nitride/Silicon dioxide bed over (100) silicon substrate. The doped PS at different temperatures has been analyzed for the grain size and the shape of the clusters, employing non-contact mode Atomic Force Microscopy (AFM). The grain size of the PS remains intact without a significant change with increasing doping temperature. A substantial increase in the cluster size and its density of the grains has been observed. The cluster formation mechanism induced by thermal variation is discussed in the context of recorded AFM images. The clusters lead to PS rings comprising of grains of the size of $100 \mathrm{~nm}$.
\end{abstract}

Keywords: Doping; Polisilicon; Nanoscale; AFM; MEMS; LPCVD; Contactmode.

\section{INTRODUCTION:}

Formation of high-quality polycrystalline silicon on low cost substrates has important applications in the development of thin film solar cells, transistors, image sensors, etc. PS is most widely used as a structural material in current microdevices. Since, it is a piezoresistive material and having an excellent compatibility with matured silicon technology. The piezoresistive effect causes the resistance of the integrated resistors to vary so that they act as strain gauges. And hence, the gauge response is proportional to the applied stress. So, it is desirable to locate the gauges in more stress areas of the diaphragm membrane. Hence, PS has large scale potentials in the emerging field of micro-electro mechanical system (MEMS $)^{1,2}$. In the field of metal oxide semiconductor (MOS) based devices and circuits, PS has been a well established material for the gates ${ }^{3,4}$. More recently PS has been identified as a promising material for realizing nanostructures embedded into silicon on insulation (SOI) structures ${ }^{1}$. Owing to its increasing applications, efforts are being made to characterize the PS films particularly deposited on insulating bed using LPCVD techniques ${ }^{5,6}$.

Thin film silicon dioxide has a compressive internal stress, less stiff than other thin film materials, has unique electrical properties and used as a mechanical material in high sensitivity applications. Silicon dioxide, with its low thermal conductivity, is a natural thermal insulator, a property which has been exploited for the production of integrated thermal detector. In MEMS, it is used to electrically isolate components and has been used in some recent applications as a structural material ${ }^{7}$.

Silicon nitride is a material that is employed in a variety of applications. Since it does not react well with many etching solutions, silicon nitride is often used to prevent impurity diffusion and ionic contamination. One of the unfortunate properties of silicon nitride is that it is not as good insulator as silicon dioxide. With a bandgap $40 \%$ smaller than $\mathrm{SiO}_{2}$, the electrical isolation provided by silicon nitride is significantly less than that of silicon dioxide. $\mathrm{Si}_{3} \mathrm{~N}_{4}$ forms a low energy barrier towards silicon, which facilitates the injection of holes into the substrate. Due to these concerns, some designers like to form most of an insulator with $\mathrm{SiO}_{2}$ and then seal its surface with silicon nitride ${ }^{8}$.

Control of the electrical conductivity of the PS layers is carried out using doping of compatible specie such as boron for ptype. However doping distribution of the specie does not follow the way it is characterized in single crystalline silicon. Effect of grain boundaries on the doping distribution in the PS layers has been studied with findings related to doping species confining to the edges of the grains ${ }^{9}$. Recently interest has been grown for analyzing the grain size of the PS films and its impact on the device characteristics ${ }^{9,10,11}$. Thermal conductivity is another parameter which carries importance due to its inherent association with the grain size of the PS layer ${ }^{12,13}$.

In the present work, LPCVD, PS film has been realized on the bed of composite layer of silicon nitride and silicon dioxide 
over (100) silicon substrate. The PS film is doped using thermal diffusion of boron at four different temperatures to adjust the sheet resistivity (SR). The thermally treated PS film is analyzed for its topological details using non-contact mode AFM in the atmospheric pressure. The grain size of the PS film has been analyzed with varying temperatures in the presence of boron doping.

The details of the experiment, carried out, are presented in the next section. The images of the grains and the clusters observed using AFM is analyzed with relevant discussion. The work is concluded at the end.

\section{EXPERIMENTAL PROCEDURE:}

Starting from a p-type (100) silicon wafer, thermal oxide was grown for a thickness of $0.5 \mu \mathrm{m}$ using conventional dry-wetdry ${ }^{14}$ procedure in a quartz furnace. Silicon nitride was then deposited over the silicon oxide bed using LPCVD, at $780^{\circ} \mathrm{C}$, for a thickness of $0.15 \mu \mathrm{m}$. The thickness of the composite layers was crucial to ensure for the strain balance at the interface of the silicon oxide and silicon nitride ${ }^{15}$. PS was deposited on the silicon nitride bed using LPCVD at $620^{\circ} \mathrm{C}$ in a furnace with a Silane $\left(\mathrm{SiH}_{4}\right)$ flow rate of $50 \mathrm{~cm}^{3} / \mathrm{min}$ and a process pressure of 0.3 torr. The thickness of the PS film was measured of $0.5 \mu \mathrm{m}$ using Nanospec Optical Spectrophotometer. Boron doping of the PS film was carried out in a furnace at four different temperatures of $920^{\circ} \mathrm{C}, 970^{\circ} \mathrm{C}$, $1020^{\circ} \mathrm{C}$ and $1070^{\circ} \mathrm{C}$, for 40 minutes in nitrogen ambient with conventional flow rate ${ }^{16}$. The borosilicate glass (BSG) invariably deposited on the PS, was etched out in buffered HF. The wafers with doped PS film at four different temperatures were oxidized at $850^{\circ} \mathrm{C}$ for 10 minutes in steam. After buffered HF treatment, all the four wafers were annealed at $920^{\circ} \mathrm{C}$ for 30 minutes in nitrogen ambient.

\section{RESULTS AND DISCUSSION:}

Etching silicon strongly depends on crystal orientation so that it follows the property of anisotropy. Various experimental techniques have been developed to study anisotropic etching of silicon. Etching can be done with various etchants with its wet chemical etchants. Several etchants are used in practice, but the most common is a strong aqueous alkali solution, e.g., $\mathrm{KOH}$. The reason is that it is less dangerous than other, easy to handle, readily available and etches fast as shown in results in fig 1 . Although it etches fast compared to other etchants, it produces poor surface finish can become covered with irregularities such as microscopic pyramids as shown in figure 2 and 3 below. The advantage of this process has been found in its simplicity and its flexibility, so that it can be used to design nowadays a wide range of microstructures and devices with fraction of micron resolution ${ }^{16,17}$. The dissolution of silicon using solution is needed for deep etching and micromachining, shaping and cleaning for research and manufacturing related to silicon devices, circuits and system often relies on wet chemical etching. Etch rate of the crystalline silicon for the given temperature and composition of etchant is not identical along all crystal planes. Results are displaying in graphical form as in fig 1 . So, anisotropy in etch rate (ERdistance traveled by the moving surface per unit time) depends on etchant's temperature ${ }^{18,19}$ and etchant composition as well as level of doping of the silicon substrate ${ }^{18}$. A high etching rate is generally desirable in a manufacturing environment. Too high etch rate however may render a process difficult to control because during etching, hydrogen bubbles $^{19}$ are formed on the silicon surface. Thus, pyramid formation ${ }^{20,21,22}$ can be related to poor bubble detachment, precluding diffusion to specific point on the surface. However, anisotropic etching has been provided as a tool for shaping of silicon structures and as an evaluation of protective surface films on silicon substrate. Due to diverse functional dependence of ER, a complete study of this system is extremely complicated. So, accuracy of etch rate may vary from laboratory to laboratory and level of measurement techniques. In this study, ER of silicon by AFM has been discussed.

During the etching process, the surface of silicon can be both hydrogen and hydroxyl-terminated, depending on the concentration and $\mathrm{pH}$ value of the etchant ${ }^{23}$. Generally, hydroxyl ions are in the form of the bubbles. In the process, they form layers which resist the etching process. Keep this in mind, a technique should follow which helps a significant fraction of the hydroxyl ions should be terminated from the surface sites ${ }^{24}$, providing the mechanism for further etching. Due to the weakening effect of the $\mathrm{OH}$ terminations there may be formation of the dragling bonds ${ }^{25}$. Thus, during the evolution of the etching process is expected that the densities of said bonds are strongly correlated with the amount of $\mathrm{OH}$ coverage. However, this correlation need not to be linear, since the weakening of covalent bonds is not the only mechanism involved in the process. The geometrical restrictions to the attachment of $\mathrm{OH}$ groups must also be taken into account. In this way, a non-monotonic dependence of the etch rate on coverage is expected for any crystallographic orientation. As the coverage increases, the etching process becomes faster, since more and more $\mathrm{OH}$ groups provide larger amounts of weaker bonds, which are more susceptible for thermal breaking. However, as the $\mathrm{OH}$ coverage is increased further, the existence of significant $\mathrm{OH} / \mathrm{OH}$ interactions between the increasing numbers of terminating hydroxyls eventually slows down the etching process. Since the process which destroys hydroxyl terminations produces hydrogen and oxygen gases. Oxygen gas is soluble in wet etchant water where as hydrogen gas is light, it evaporates ${ }^{26}$. At the same time recombination process may also occurs which ultimately urged the etch rate become vanishingly small for the limiting cases of complete hydrogen and complete hydroxyl termination (complete passivation). Therefore, for any crystallographic orientation, there should exist a value of coverage by which the etch rate reaches a maximum. One of the purposes of this work is to show that, in spite of the simplicity of the previous reasoning, the existence of a maximum etch rate as a function of coverage. 

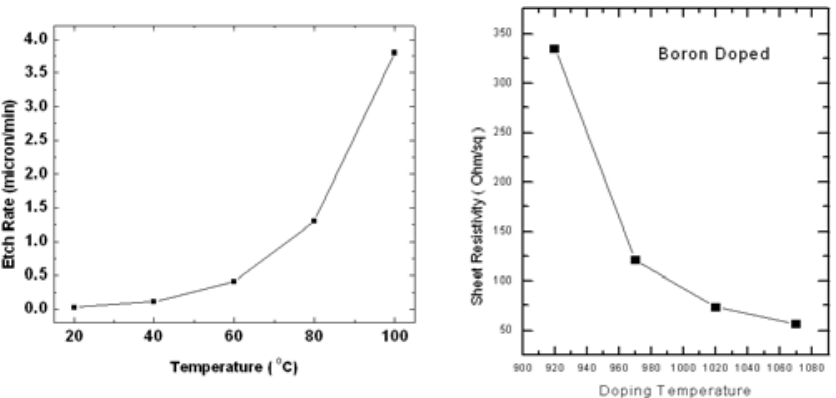

Figure-1: Variation of ER and SR for boron doped PS with temperatures

Thin PS film is deposited on silicon substrate may have residual stress. During deposition, residual stress can be controlled entirely by varying deposition pressure and temperature. Thus mech2anical property of PS film depends greatly upon the process used in deposition. In this study attention is being taken appreciably.

The films exhibit preferential grain orientations as shown in fig 2 should vary with temperature. Since an ideal film does not exhibit orientation dependence for its mechanical properties. Researchers have found that depositing films at $590^{\circ} \mathrm{C}$, which is the transition point between polycrystalline and amorphous silicon, is an effective method of producing an isotropic film of PS. At this temperature amorphous silicon will recrystalizes during annealing and produces film with nearly uniform Young's modulus.

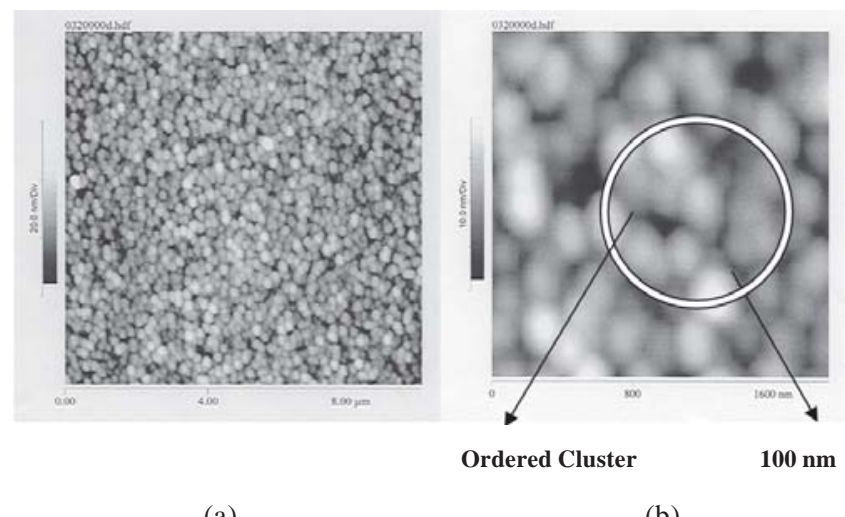

(a)

(b)

Figure-2: AFM Micrograph of PS grains doped at $1070{ }^{\circ} \mathrm{C}$. (a) Scan area of $(10.0 \mu \mathrm{m} \times 10.0 \mu \mathrm{m})$ (b) scan area of $(1.5 \mu \mathrm{m} \times 1.5 \mu \mathrm{m})$.

The PS films, doped separately with boron at $920^{\circ} \mathrm{C}, 970^{\circ} \mathrm{C}$, $1020^{\circ} \mathrm{C}$ and $1070^{\circ} \mathrm{C}$ were electrically characterized by measuring the sheet resistivity (SR) using four point probe method ${ }^{27}$. Fig.1 shows the variation of the sheet resistivity of the PS layers with varying temperature. As a thumb rule, PS sheet resistivity doubles that of crystalline silicon at equivalent doping conditions ${ }^{28}$. The trend of the SR variation and the numerical values ensure the complete removal of the borosilicate glass from the PS. Beyond critical temperature, PS film starts crystallization and form special kind of geometrical structure in the form of grains. The sizes of grains are no more than film thickness. AFM in non-contact mode was employed to analyse the grains of the PS layer. The AFM micrographs of the PS films doped at $1070^{\circ} \mathrm{C}$ have been shown in the Fig.2. that shows the evolution of grains with tempera- ture. The AFM micrograph confirms the polycrystalline nature of the doped film. As we have seen, grains are of more or less equal size with said temperature. Thus, doping temperature does not affect the grain size of the PS as is evident from Fig. 2(a). Careful examining of the micrographs indicates, aligning of the grains in the shape of rings. The density of the grains in one ring grows further with increasing doping temperature. Fig. 2(b) show the enlarged version of the micrographs for PS layers doped at $1070^{\circ} \mathrm{C}$. Based on the micrograph, the average size of the grains is $100 \mathrm{~nm}$ which confirms grain size invariance with doping temperature. Generally, variation of grain size in the PS film with temperature has been considered as a common phenomenon ${ }^{29}$. However, in the present case stability in the grain size with varying temperature and in the presence of boron doping is a key point that focused our attention. To explain such interesting findings, focus is being given for fundamental understanding of invariance of grain structure with annealing.

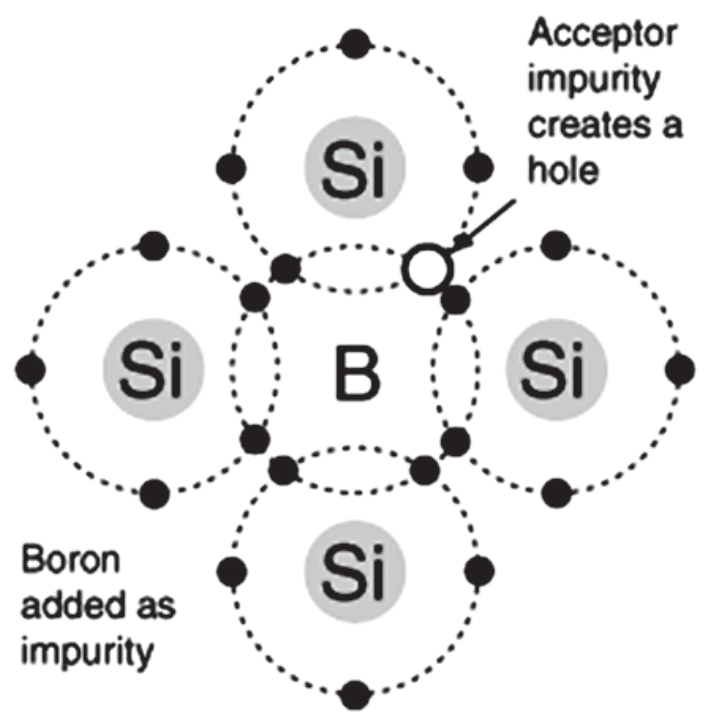

Figure-3: Formation of boron doped silicon crystal

As boron is doped into silicon there is deficiency of one electron, i.e. hole, in comparison with pure silicon. The valence band of doped silicon is therefore no longer completely full. Thus, under the influence of thermal energy electron can change its state from valance band to conduction band. This kind of effect accumulated in solid resulted into a flood of the carrier and is better known as flow of current. The mechanism of this type of conductivity is p-type. Consider an electronic state of wave vector ' $k$ ' near the edge of the valence band, and with the energy less than that associated with the band edge. The energy difference, $\Delta E$, is

$$
\Delta E=\frac{1}{2 m_{v}}\left[\hbar\left(k-k_{0}\right)\right]^{2}
$$

When an electron is in a state within the edge of the valence band, it wouldn't be free, because, there is a strong interaction between the electron wave and the lattice vibration, so that a kind of a force is developed which causes it to acceler- 
ate in the opposite direction. The movement of an electron on the top of the almost full band is equivalent to the movement of a positive hole at the bottom of the band. Hence, a hole is liberated by thermal agitation and delocalized in the lattice. In this way, the existence of p-type conductivity is an essential feature in physics and technology.

With little elaboration, we can take Wiedemann-Franz law ${ }^{13}$ in modified form as:

$\frac{K}{\sigma T}=\frac{\pi^{2} k_{B}^{2}}{3|e|^{2}}$, Where $K$ is thermal conductivity and $\sigma$ is p-type conductivity.

$$
\sigma=n|e| \mu_{h}=2\left(\frac{m k_{B} T}{2 \pi \hbar^{2}}\right)^{\frac{3}{2}} e^{-\left(\frac{E_{g}}{2 k_{B} T}\right)} \cdot|e| \mu_{h}
$$

This suggests that in PS film, holes are responsible for transport of heat as well as conduction. Since, above absolute zero, there is gradual increase in the amplitude of atomic vibration that influences lattice. Thus, transverse and longitudinal phonons are generated during the process. Part of these phonons is responsible for thermal conductivity where as remarkable fraction of phonons should be involved to electrical conductivity as described elsewhere in this paper.

It is recognize that Fermi level marks the dividing line, in energy, between whether state is more probably occupied or more probably empty. The scattering of electron by vibrating ions in a solid at a temperature $T$, the energy transfer between the electron and the ion is in the order of $k T$. If an electron is in a state with energy below the Fermi energy, the probability that such a state is occupied is essentially one. The probability of an unoccupied state with same energy is essentially zero. There are many such electrons since the probability that such a state is occupied is essentially one and the probability of an unoccupied state with energy close to this value is essentially zero. Because, Pauli Exclusion Principle prohibits more than a couple of electron having opposite spin occupying a state. Thus there is no available state as well as energy exchange between electrons inside the Fermi sphere since all available electron states are already occupied. This may be the probable reason why grain size remains invariance with thermal agitation in boron doped polysilicon film.

Another interesting observation in Fig.2 (b) is that clustering of the grains in the shape of rings and the increasing grain density in each cluster with increasing temperature. This might be due to the presence of boron doping at high temperature annealing. Density of states of boron may arrive at saturation at high temperature annealing. It equally affects not only microstructure but also in electrical properties also. It has been analyzed that the electrical conductivity of the PS film is controlled by the boron doping profile and does not vary due to grains or clustering of the grains ${ }^{30}$. Because of the charge neutrality principle within the atom, electrons within the different shells of the boron have a tendency of diffusion inside the grains. The ordered clustering of the PS grains seems attractive as it leads to nano-scale entity which could be realized by reducing the grain size during LPCVD. The present work advocates a doping specie dependent phenomenon at annealing temperatures in the LPCVD, PS film resulting in to stabilize grain size with associated ordered clustering of the grains which grows further with increasing temperature. Hopefully, saturation takes place beyond the certain critical temperature.

\section{CONCLUSION:}

In conclusion, during the temperature evolution with silicon, formation of a solvable silicon complex is the first substitution of terminating hydrogen by a hydroxyl group. After this substitution, the sequence of further chemical steps is fast. Therefore, it can be expected that termination of the hydrogen from neighboring atoms of initially $\mathrm{OH}$-terminated sites will occur shortly after the initial substitution, before detachment of the solvable silicon complex. Experimental observation by AFM of aligning of the grains in a shape of ring like cluster has been shown in LPCVD, PS film on Silicon nitride. Temperature dependent growth of the PS rings with no change in the grain size and stipend conductivity provides a new front in the area of nanotechnology. The technology seems to be competent to realize nanoscale structures in SOI environment. It is therefore important to establish to what extent the existence of clustering affects the etch rate and the anisotropy of the etching process.

\section{ACKNOWLEDGEMENTS}

Thanks for J. Akhtar, Scientist CEERI, Pilani, for helping sample preparation. Thanks for School of Physical Sciences, JNU to allow author to perform AFM experiment. Thanks for UGC, Kathmandu, for financial support.

\section{REFERENCES}

1. Binnig, G., Rohrer, H., Gerber, C. \& Weibel, E. 1982. Surface studies by scanning tunneling microscopy. Phys. Rev. Lett. 49: 57-61.

2. Eigler, D. M. \& Schweizer, E. K. 1990. Positioning single atoms with a scanning tunnelling microscope. Nature 344: 524-526.

3. Lyo, I.-W. \& Avouris, P. 1991. .Field-induced nanometer- to atomic-scale manipulation of silicon surfaces with the STM. Science 253: 173-176.

4. Eigler, D. M., Lutz, C. P. \& Rudger, W. E. 1991. |An atomic switch realized with the scanning tunnelling microscope. Nature 352: 600-603.

5. Terabe, K., Hasegawa, T., Nakayama, T. \& Aono, M. 2005. Quantized conductance atomic switch. Nature 433: 47-50.

6. Nilius, N., Wallis, T. M. \& Ho, W. 2002. Development of onedimensional band structure in artificial gold chains. Science 297: 1853-1856.

7. Nazin, G. V., Qiu, X. H. \& Ho, W. 2003. Visualization and spectroscopy of a metal-molecule-metal bridge. Science 302: 77-81.

8. Repp, J., Meyer, G., Paavilainen, S., Olsson, F. E. \& Persson, M. 2006. Imaging bond formation between a gold atom and pentacene on an insulating surface. Science 312: 1196-1199.

9. Lafferentz, L. et al. 2009. |Conductance of a single conjugated polymer as a continuous function of its length. Science 323: 1193-1197. 
10. Heinrich, A. J., Lutz, C. P., Gupta, J. A. \& Eigler, D. M. 2002. |Molecule cascades. Science 298: 1381-1387.

11. Crommie, M. F., Lutz, C. P. \& Eigler, D. M. 1993. Confinement of electrons to quantum corrals on a metal surface. Science 262: $218-220$.

12. Heller, E. J., Crommie, M. F., Lutz, C. P. \& Eigler, D. M. 1994. Scattering and adsorption of surface electron waves in quantum corrals. Nature 369: 464-466.

13. Manoharan, H., Lutz, C. P. \& Eigler, D. M. 2000. Quantum mirages formed by coherent projection of electronic structure. Nature 403: 512-515.

14. Moon, C. R., Mattos, L. S., Foster, B. K., Zeltzer, G. \& Manoharan, H. C. 2009. Quantum holographic encoding in a two-dimensional electron gas. Nature Nanotech. 4; 167-172.

15. Kitchen, D., Richardella, A., Tang, J.-M., Flatté, M. E. \& Yazdani, A. 2006. Atom-by-atom substitution of Mn in GaAs and visualization of their hole-mediated interactions. Nature 442: 436-439.

16. Yamachika, R., Grobis, M., Wachowiak, A. \& Crommie, M. F. 2004. Controlled atomic doping of a single $C_{60}$ molecule. Science 304: 281-284.

17. Hla, S.-W., Bartels, L., Meyer, G. \& Rieder, K.-H. 2000. Inducing all steps of a chemical reaction with the scanning tunneling microscope tip: Towards single molecule engineering. Phys. Rev. Lett. 85: 2777-2780.

18. Jung, T. A., Schlittler, R. R., Gimzewski, J. K., Tang, H. \& Joachim, C. 1996. Controlled room temperature positioning of individual molecules: Molecular flexure and motion. Science 271: 181-184.

19. Gimzewski, J. K. et al. 1998. Rotation of a single molecule within a supramolecular bearing. Science 281: 531-533.

20. Stipe, B. C. \& Ho, W. 1998. Inducing and viewing the rotational motion of a single molecule. Science 279: 1907-1909.

21. Komeda, T., Kim, Y., Kawai, M., Persson, B. N. J. \& Ueba, H. 2002. Lateral hopping of molecules induced by excitation of internal vibration mode. Science 295: 2055-2058.

22. Pascual, J. I., Lorente, N., Song, Z., Conrad, H. \& Rust, H.-P. 2003. Selectivity in vibrationally mediated single-molecule chemistry. Nature 423: 525-528.

23. Lee, H. J. \& Ho, W. 1999. Single-bond formation and characterization with a scanning tunneling microscope. Science 286: $1719-1722$.

24. Chen, W., Jamneala, T., Madhavan, V. \& Crommie, M. F. 1999. Disappearance of the Kondo resonance for atomically fabricated cobalt dimers. Phys. Rev. B 60: R8529.

25. Hirjibehedin, C. F., Lutz, C. P. \& Heinrich, A. J. 2006. Spin coupling in engineered atomic structures. Science 312: 1021-1023.

26. Giessibl, F. J. \& Quate, C. F. 2006. Exploring the nanoworld with atomic force microscopy. Physics Today 59: 44-50.

27. Giessibl, F. J. 2003. Advances in atomic force microscopy. Rev. Mod. Phys. 75: 949-983.

28. García, R. \& Pérez, R. 2002. Dynamic atomic force microscopy methods. Surf. Sci. Rep. 47: 197-301.

29. Binnig, G., Quate, C. F. \& Gerber, C. 1986. Atomic force microscope. Phys. Rev. Lett. 56: 930-933.

30. Gerber, C. \& Lang, H. 2006. How the doors to the nanoworld were opened. Nature Nanotech. 1: 3-5. 